\title{
Optimization of Competitiveness in Sports Swimming
}

\author{
Kubeev A.V. \\ Federal Scientific Center Institute \\ Moscow, Russia \\ 1574373@mail.ru
}

\author{
Mochalova M.S. \\ Federal Scientific Center Institute \\ Moscow, Russia \\ m.mochalova80@mail.ru
}

\author{
Gonzalez S.E. \\ Department of Physical Education and Sports \\ Peoples Friendship University of Russia (RUDN) \\ Moscow, Russia \\ vniifk@yandex.ru
}

\begin{abstract}
Researching the materials of official protocols of four world swimming championships over a period of 2013-2019 provided grounds for optimization of competitiveness quantitative and qualitative criteria in sports swimming. The method is offered concerning the differentiation of athletes' general sample pattern structure by each swimming specialization. The unequal density of sports results in the ranges of linear and non-linear distribution is determined, which affects the quality of the results analysis in swimming. The highest density of the results distribution function, with the corresponding number of high-level athletes, regarding sports and technical readiness, is the optimizing factor of their high competitiveness in sports contests. The optimal algorithm of calculating parameters of results distribution and quantitative structure of various samples of athletes of each swimming specialization is developed and tested. The implementation of an integrated approach to optimizing the analysis of the distribution function generates a more accurate forecast of results in various disciplines of swimming based on the application of standard criteria.
\end{abstract}

Keywords—sports result; results sampling; group of athletes; swimming specialization; optimization method; distribution function; linearity-nonlinearity of distribution.

\section{INTRODUCTION}

Modern rates of high achievements sports development characterize large-scale expansion of competitive activity of athletes, including sports swimming.

One of the priority scientific directions of research in sports of high achievements, from methodological point of view, was the optimization of parameters of training and competitive activity of athletes.

\section{LITERATURE REVIEW}

However, studies on swimming over the past two decades have paid very little attention to these issues. In relation to the level of high sports achievements, there is an increased interest of specialists and athletes to the issues of providing technologies for sports improvement $[1,2,3,4,5,6]$, an expanded search for reserves to improve results in competitions of different levels $[7,8,9,10]$ at different distances of sports swimming [11,12,13,14,15,16,17].

The relevance of this study is determined by the need to expand the scientific search in the context of the issue of optimizing the analysis of competitive activity of high-class athletes. In this regard, a scientifically and practically justified search method for an optimal solution to this problem is necessary.

The purpose of the study was determined with regard to the need to optimize and improve the methods of analyzing the main criterion of success in sports - the results of sports activities.

\section{RESEARCH METHODOLOGY}

In the most general form, the solution of the first optimization problem was to provide information analysis of the distribution function of the highly qualified swimmers results, which assumed proper arrangement of the criteria and definition of the initial data system format. The presentation of the athletes' results in accordance with the occupied places definitely determined the format of rank distribution of the average swimming speed values (Vaver.) at the distance for each swimming style.

The study and analysis of the results distribution of sports swimming competitions was carried out on the basis of official data in the protocols of four world cup championships (FINA) over a period of 2013-2019. The analysis technology provided proper arrangement of initial data in a required sequence of the swimmers activity most informative values indicators in preliminary heats, semi-finals and finals. It is well known that the implementation of this technique involves 
arrangement of the initial data and the presence of substantive and computational components of the analysis. As the main carriers of the required information were identified the following characteristics - average speed value of covering the distance (Vaver.), standard parameters of linear approximation of the values distribution function (Vaver.), as well as values of angular (a) and linear $(b)$ coefficients of the results distribution function and the coefficient of determination $\left(R^{2}\right)$. The calculated result of the conditional leader $\left(V_{1}\right)$ was used as a short-term forecast criterion.

Thus, for the effective analysis of the initial and basic design characteristics of competitive activity it was necessary "to adjust" the primary information logically and structurally and in the same way to assess the possible results of intermediate operations.

In the distribution of individual average speed values in general groups of athletes, regardless of swimming style, there was as a rule an alternation of results linear and nonlinear distribution sections. The ranges of linear distribution were characterized by minimal differences in results, that is, they indicated a high level of competitiveness (special training) of the leading athletes. Various variants and features of the sports results distribution were presented in the format of a linear function, as well as in the formats of power polynomials (for example, $2^{\text {nd }}$ or $3^{\text {rd }}$ degree), for the purpose of comparative evaluation of approximation methods. These well-known methods have been widely used in previous studies of this type.

To improve the efficiency of the analysis, it is advisable to present the distribution function of sports results from the standpoint of the athletes' total sample heterogeneity in each discipline of swimming.

In this regard, the solution of the second problem optimization of the athletes composition in each general group of specialization in swimming - assumed the use of a differentiated approach to determining the quantitative ratio of athletes in groups of different categories, in accordance with the status of their sports readiness (group "leaders"), their closest competitors (group "main") and athletes from the bottom of protocols lists (group "outsiders").

During identification of groups, we relied on characteristic features of athletes representing them. First, the nonlinear nature of the "leaders" group athletes average distance speed distribution values is generally conditioned by obvious advantage of a small number of the most prepared athletes. Second, the specificity of the "main" group was considered in the context of constant competition with the "leaders" group, regardless of the swimming style. The number of athletes, competing in the "leaders" and "main" groups, totally was no less than $50-60 \%$ of the whole number of competitors in different styles of swimming. Therefore, the division of the "main" group into two subgroups $(A$ and $B$ ) generally is not required, but it is necessary in large samples. Third, a significant decrease in average distance speed, regardless of the swimming style, objectively indicated an insufficient level of weaker athletes (group "outsiders") special training.
Thus, the study used two main methods to optimize the analysis of the results. First, the method of identifying features of the distribution function of sports results in general and specific athletes' groups of different specializations (Vaver.). Secondly, we applied a differentiated method of determining the quantitative composition of athletes total samples for each distance and swimming style.

\section{RESULTS}

The preliminary study of the four world swimming championships (2013-2019) results showed that, due to the specific features of highly qualified athletes samples, the level of their relative competitiveness is usually heterogeneous in most competitions, according to the criterion of decreasing the distribution function values (Vaver.). A noticeably different character of Vaver. distribution in the selected groups of athletes testified to the presence of the samples specific features. The evident nature of their demonstration is clearly visible in each discipline of sports swimming, especially on the opposite "edges" of the total sample of athletes of the swimming specialization (leaders and outsiders). Therefore, in the analysis of these specific ranges of Vaver. distribution, it is advisable to use other schemes of calculated coefficients, based on complex mathematical methods (which are not considered here).

However, quite often the problems of sports practice can be solved in a relatively simple, but more rational way of processing the Vaver. distribution function, for example, in the alternating ranges of linear and nonlinear sections of the Vaver. distribution function. In such variants of Vaver. values distribution, the application of iterations methods (consecutive approximations) and approximation of initial data by "sensitive" power polynomials allows to study sequentially the above mentioned areas of Vaver. distribution in the total sample of athletes. Below is a typical example (Fig. 1).

In the "leaders" zone, the results of the eight best athletes participating in the final heats were visually distributed. The dependence line in this section of the diagram is markedly different from the general trend of the Vaver. distribution function. At the same time, as can be seen from this diagram, a more detailed representation of the results distribution function is possible, which gives a refinement of the function values in favor of "leaders" - from the first to the fourth places. However, the values of angular coefficients $(a)$ of linear functions in both sections of the athletes-leaders results distribution and the main group results differed from each other four times. In this regard, it is necessary to note the cases when the participants of the finals, who took the last places in the end, showed their worst results compared to the previous stage of the competition.

The results of this study also revealed the following features of the demonstration of sports results distribution in certain groups of athletes, but within a specific swimming specialization.

For example, in all styles of high-speed swimming (at distances of 50 and 100 meters by different swimming styles), sportswomen of the main group A had the best averaged values of angular coefficient $(a)$. Its relatively best values 
ranged from -0.0023 to -0.0027 in breaststroke and freestyle respectively. At the lower limit, the range of variation in the values of angular coefficient in the same group was from 0.0031 to -0.0046 in backstroke and breaststroke respectively. At sprint distances, slightly "lagging" values of angular coefficient in any swimming style can be explained by the influence of not quite successful execution of the start.

The regularity of another type can be distinguished by changes in the values of linear coefficient $(b)$ of the results distribution in groups of the female athletes' general sample according to the swimming styles. In essence, the values of this coefficient are close to the forecast - the calculated average distance speed of the conditional leader Vaver (1). These data show that the value of this coefficient decreases in accordance with the average level of athletes' preparedness. So, for example, in "the fastest" freestyle the lag of the main group A sportswomen from the leaders group by the value of linear coefficient was $4.12-4.64 \%$; in "the slowest" breaststroke - was almost the same, 4.05-4.68\%. Accordingly, the lag of athletes of the main group B from the main group A by the value of linear coefficient was already $4.60-5.34 \%$ in freestyle and up to $5.49 \%$ - in breaststroke.

At the distance of $100 \mathrm{~m}$ by various swimming styles, the following results of sportswomen in different groups as part of the general group of swimming specializations were obtained. Just as in the $50 \mathrm{~m}$, the representatives of the main group A had the best averaged values of angular coefficient $(a)$ in all styles of high-speed swimming. Its relatively best values ranged from -0.0022 to -0.0034 in backstroke and freestyle respectively. At the lower limit, the range of variation in the values of angular coefficient in this group was from -0.0029 to -0.0044 in backstroke and breaststroke respectively.

Judging by dynamics of linear coefficient $(b)$ it was possible to observe that value of coefficient decreases according to the level of sportswomen preparedness. At a 100meter freestyle distance, as well as at a 50-meter distance, lag of sportswomen of the main group A from the group of leaders by value of linear coefficient was $2.92-3.99 \%$ on the average. In butterfly, this figure reached $4.90 \%$, in breaststroke was slightly lower $-3.56 \%$. Accordingly, the lag of sportswomen of the main group B from the main group A in terms of the linear coefficient was $4.71 \%$ in freestyle, $5.40 \%$ in butterfly and reached its maximum values in breaststroke, 6.29-6.35\% upon the average.

Similar trends in the dynamics of the average distance characteristic parameters velocity distribution were observed in different sample groups of male athletes.

Basing on the above mentioned and other examples (not given here), we applied the following preliminary version of the algorithm for processing the results when analyzing the results of swimming competitions:

1) to select in each general group of $50 \%$ of athletes who have completed the swimming distance;

2) to eliminate the top 5 results at each swimming distance;
3) to make a regression equation, required for calculating the values of angular and linear coefficients, for the remaining number of athletes at each distance;

4) to determine by the angular coefficient the level of athletes' relative competitiveness using comparative analysis with other competitions;

5) to calculate using the regression equation the value of average distance velocity $(Y)$ of the calculated, conditional leader at $X=1$.

Processed data of the 2013-2019 swimming world cups results, according to the specified algorithm " $50 \%$ minus 5 best times", are represented in tables I and II.

TABLE I. PARAMETERS OF COMPETITIVE ACTIVITY IN SWIMMING BASED ON THE RESULTS OF 2013-2019 WORLD CHAMPIONSHIPS (FREESTYLE, WOMEN)

\begin{tabular}{|c|c|c|c|c|c|c|}
\hline \multirow{2}{*}{$\begin{array}{c}\text { Distance } \\
\mathrm{m}\end{array}$} & \multicolumn{3}{|c|}{2013} & \multicolumn{3}{|c|}{2015} \\
\hline & $\mathrm{a}$ & b & $\begin{array}{c}\text { Vaver.(1), } \\
\mathrm{m} / \mathrm{s}\end{array}$ & $\mathrm{a}$ & b & $\begin{array}{c}\text { Vaver. (1) } \\
\mathrm{m} / \mathrm{s}\end{array}$ \\
\hline 50 & -0.004 & 2.045 & 2.041 & -0.003 & 2.037 & 2.034 \\
\hline 100 & -0.003 & 1.870 & 1.867 & -0.003 & 1.859 & 1.856 \\
\hline 200 & -0.003 & 1.738 & 1.735 & -0.003 & 1.717 & 1.714 \\
\hline 400 & -0.005 & 1.661 & 1.657 & -0.003 & 1,628 & 1.624 \\
\hline 800 & -0.003 & 1.600 & 1.597 & -0.004 & 1.590 & 1.586 \\
\hline 1500 & -0.005 & 1.593 & 1.588 & -0.006 & 1.566 & 1.559 \\
\hline \multirow{2}{*}{$\begin{array}{c}\text { pistance } \\
\mathrm{m}\end{array}$} & \multicolumn{3}{|c|}{2017} & \multicolumn{3}{|c|}{2019} \\
\hline & $\mathrm{a}$ & b & $\begin{array}{c}\text { Vaver. (1) } \\
\mathrm{m} / \mathrm{s}\end{array}$ & $\mathrm{a}$ & b & $\begin{array}{c}\text { Vaver. (1) } \\
\mathrm{m} / \mathrm{s}\end{array}$ \\
\hline 50 & -0.004 & 2.051 & 2.047 & -0.005 & 2.080 & 2.076 \\
\hline 100 & -0.004 & 1.886 & 1.882 & -0.004 & 1.909 & 1.906 \\
\hline 200 & -0.004 & 1.729 & 1.726 & -0.004 & 1.742 & 1.739 \\
\hline 400 & -0.005 & 1.633 & 1.629 & -0.004 & 1.650 & 1.646 \\
\hline 800 & -0.004 & 1.580 & 1.579 & -0.005 & 1.615 & 1.611 \\
\hline 1500 & -0.004 & 1.547 & 1.543 & -0.006 & 1.608 & 1.602 \\
\hline \multirow{2}{*}{\begin{tabular}{|} 
pistance \\
$\mathrm{m}$
\end{tabular}} & \multicolumn{6}{|c|}{ Average values over a period of 2013-2019 } \\
\hline & \multicolumn{3}{|c|}{$\mathrm{a}$} & \multicolumn{3}{|c|}{ Vaver.(1), $\mathrm{m} / \mathrm{s}$} \\
\hline 50 & \multicolumn{3}{|c|}{-0.0040} & \multicolumn{3}{|c|}{2.0492} \\
\hline 100 & \multicolumn{3}{|c|}{-0.0032} & \multicolumn{3}{|c|}{1.8777} \\
\hline 200 & \multicolumn{3}{|c|}{-0.0033} & \multicolumn{3}{|c|}{1.7283} \\
\hline 400 & \multicolumn{3}{|c|}{-0.0041} & \multicolumn{3}{|c|}{1.6389} \\
\hline 800 & \multicolumn{3}{|c|}{-0.0037} & \multicolumn{3}{|c|}{1.5926} \\
\hline 1500 & \multicolumn{3}{|c|}{-0.0053} & \multicolumn{3}{|c|}{1.5732} \\
\hline
\end{tabular}

\section{CONCLUSIONS}

1. The format of rank values distribution of average speed of covering a sprint distance (Vaver.), irrespective of the swimming style, is objectively optimum when studying the results distribution function of highly-qualified athletes. This conclusion comes from the logic of competitive activity absolute and relative characteristics complex assessment 
basing on differentiation of the athletes' general sample separate groups in various specializations of swimming.

2. The results of the study determined that the highest density of the results distribution function (Vaver.) with the corresponding number of high-level athletes, regarding their sports and technical readiness, is the optimizing factor of their high competitiveness in sports contests.

3. The approved algorithm for calculating the parameters of the results distribution and the quantitative composition of the athletes' total sample of each swimming specialization seems to be a necessary component of sports results distribution function optimization.

4. Additional verification calculations showed that the results of the studies are dependent on random or intentional changes in the parameters of each studied sample of athletes. Differences in grades can reach 5-6 percent, which is significant for each swimming specialization. In this regard, a clear justification of quantitative and qualitative criteria for the formation of the highly qualified athletes studied samples is relevant.

TABLE II. PARAMETERS OF COMPETITIVE ACTIVITY IN SWIMMING BASED ON THE RESULTS OF 2013-2019 WORLD CHAMPIONSHIPS (FREESTYLE, MEN)

\begin{tabular}{|c|c|c|c|c|c|c|}
\hline \multirow{3}{*}{$\begin{array}{c}\text { Distance, } \\
\mathrm{m}\end{array}$} & & & & & & \\
\hline & \multicolumn{3}{|c|}{2013} & \multicolumn{3}{|c|}{2015} \\
\hline & $a$ & $b$ & $\begin{array}{c}\text { Vaver. (1), } \\
\mathrm{m} / \mathrm{s}\end{array}$ & $a$ & $b$ & $\begin{array}{c}\text { Vaver. (1), } \\
\mathrm{m} / \mathrm{s}\end{array}$ \\
\hline 50 & -0.004 & 2.304 & 2.300 & -0.004 & 2.285 & 2.282 \\
\hline 100 & -0.003 & 2.076 & 2.072 & -0.002 & 2.067 & 2.065 \\
\hline 200 & -0.002 & 1.874 & 1.872 & -0.002 & 1.883 & 1.881 \\
\hline 400 & -0.003 & 1.764 & 1.761 & -0.002 & 1.767 & 1.765 \\
\hline 800 & -0.003 & 1.701 & 1.698 & -0.003 & 1.718 & 1.716 \\
\hline 1500 & -0.003 & 1.673 & 1.670 & -0.003 & 1.679 & 1.676 \\
\hline \multirow{2}{*}{$\begin{array}{c}\text { Distance, } \\
\mathrm{m}\end{array}$} & \multicolumn{3}{|c|}{2017} & \multicolumn{3}{|c|}{2019} \\
\hline & $a$ & $b$ & $\begin{array}{c}\text { Vaver. (1), } \\
\mathrm{m} / \mathrm{s}\end{array}$ & $a$ & $b$ & $\begin{array}{c}\text { Vaver. (1), } \\
\mathrm{m} / \mathrm{s}\end{array}$ \\
\hline 50 & -0.0041 & 2.358 & 2.3541 & -0.003 & 2.310 & 2.308 \\
\hline 100 & -0.0018 & 2.074 & 2.0720 & -0.002 & 2.084 & 2.082 \\
\hline 200 & -0.0016 & 1.885 & 1.8832 & -0.002 & 1.909 & 1.907 \\
\hline 400 & -0.0028 & 1.779 & 1.7761 & -0.003 & 1.796 & 1.793 \\
\hline 800 & -0.0026 & 1.7078 & 1.7042 & -0.003 & 1.732 & 1.729 \\
\hline 1500 & -0.0021 & 1.678 & 1.6754 & -0.003 & 1.700 & 1.697 \\
\hline \multirow{2}{*}{$\begin{array}{l}\text { Distance, } \\
\mathrm{m}\end{array}$} & \multicolumn{6}{|c|}{ Average values over a period of 2013-2019 } \\
\hline & \multicolumn{3}{|c|}{$a$} & \multicolumn{3}{|c|}{ Vaver. (1), $\mathrm{m} / \mathrm{s}$} \\
\hline 50 & \multicolumn{3}{|c|}{-0.0037} & \multicolumn{3}{|c|}{2.3109} \\
\hline 100 & \multicolumn{3}{|c|}{-0.0023} & \multicolumn{3}{|c|}{2.0728} \\
\hline 200 & \multicolumn{3}{|c|}{-0.0020} & \multicolumn{3}{|c|}{1.8857} \\
\hline
\end{tabular}

\begin{tabular}{|l|l|l|}
\hline 400 & -0.0027 & 1.7739 \\
\hline 800 & -0.0026 & 1.7117 \\
\hline 1500 & -0.0028 & 1.6795 \\
\hline
\end{tabular}

Thus, the results obtained in this study allow us to formulate the following conclusions.

\section{References}

[1] T. Całka, R. Muszkieta, M. Cleślicka. Analysis of results of European Swimming Championship in London 2016. Journal of Education, Health and Sport. 2016, vol. 6, 10, pp. 754-771. URL: http://doi.org/10.5281/zenodo.258831.

[2] S.V. Dustker, D.M. Nayak. Performance Analysis of Individual Swimmers for Elite Competitive Swimming. International Journal of Engineering Research And Advanced Technology (IJERAT). 2016, special vol. 02, 01, pp. 142. URL: https://ijerat.com/uploads/2/3270_pdf.pdf.

[3] M. Elipot, G. Dietrich, P. Hellard, N. Houel. A New Software for Swimming Races Analysis. $8^{\text {th }}$ Conference of the International Sports Engineering Association (ISEA) Poster Session II, July 2010. Abstracts: Procedia Engineering. 2010, pp. 3467. DOI:10.1016/j.proeng.2010.04.191. URL: https://hal-insep.archivesouvertes.fr/hal-01724273/file/70-\%20Cinalysis\%20a\%20new.pdf.

[4] B. Jorgić, M. Puletić, R. Stanković, T. Okičić, S. Bubanj, R. Bubanj. The Kinematic Analysis of the Grab and Track Start in Swimming. Facta Universitatis, Series: Physical Education and Sport. 2010, vol. 8, 1, pp. 31-36.

[5] F. Krüger. Analysis of kinematic parameters during competitive backstroke swimming. International Series on Sport Sciences book series (MMSS). Biomechanics IV (Palgrave, London). pp. 208-216. URL: $\quad$ https://doi.org/10.1007/978-1-349-02612-8_30, https://link.springer.com/chapter_10.1007_978-1-349-026128_30_files.

[6] A. Lavie. The swimming of the POD: Theoretical analysis and experimental results. IEEE Trans. on Magn. 1970, vol. 6, pp. 365-367. DOI: 10.1109/TMAG.1970.1066809.

[7] J. Jürimäe, K. Haljaste, A. Cicchella, E. Lätt, P. Purge, A. Leppik, T. Jürimäe. Analysis of Swimming Performance from Physical, Physiological and Biomechanical Parameters in Young Swimmers. Pediatric Exercise Sciences. 2007, vol. 19, pp. $70-81$.

[8] M.I. Love, Ch. Soneson, R. Patro. Swimming downstream: Statistical Analysis of differential transcript usage following Salmon quantification. $2018 . \quad$ URL: http://www.bioconductor.org/packages/devel/workflows/vignettes/rnas eqDTU/inst/doc/rnaseqDTU.html.

[9] B.R. Mason, J. Fowlie. Competition Analysis for high Performance swimming. In book: The AIS International Swim Seminar Proceedings, Chapter: Competition Analysis for High Performance Swimming, Publisher: Australian College of Sports Education, Editors: Australian Institute of Sport. pp. 5-18. URL: https://www.researchgate.net/publication/236235692_Competition_An alysis_for_High_Performance_Swimming.

[10] B. Radulovic. The Analysis of trend of Swimming Results in Montenegro from 2000-2009. Sport Mont. 2010, vol. VIII, pp. 219-225. URL: http://www.sportmont.ucg.ac.me/?sekcija=article\&artid=332; http://www.sportmont.ucg.ac.me/clanci/SportMont_Dec_2010_Radulo vic_219-225.pdf.

[11] A.J. Silva, A. Rouboa, A. Moreira, V.M. Reis, F. Alves, J.P. VilasBoas, D.A. Marnho. Analysis of Drafting Effects in Swimming using Computational Fluid Dynamics. Journal of Sports Science and Medicine. 2008, 7, pp. $60-66$.

[12] A.B. Ramos-Hryb, Z. Bahor, S. McCann, E. Sena, M.R. MacLeod, C. Lino de Oliveira. Protocol for a Systematic Review and Meta-analysis of Data from preclinical Studies Employing forced swimming test: an Update. BMJ Open Science. 2019, vol. 3, pp. 35. DOI: 10.1136/bmjos2017-000043. http://openscience.bmj.com/. 
[13] R. Schulz, and Ch. Curnow. Peak Performance and Age Among Superathletes: Track and Field, Swimming, Baseball, Tennis, and Golf. Journal of Gerontology. 1988, vol. 43, 5, pp. 113-120. URL: https://doi.org/10.1093/geronj/43.5.P113.

[14] W. Weimar, A. Summer, B. Romer, J. Fox, J. Rehm, B. Decoux, J. Patel. Kinetic Analysis of Swimming Flip-Turn Push-Off Techniques. Journal Sports. 2019, vol. 7, 2, pp. 32. URL: https://doi.org/10.3390/sports7020032; https://www.mdpi.com/20754663/7/2/32/htm.

[15] C. Tomasz, R. Muszkieta, M. Cieślicka. Analysis of results European Swimming Championship in London. Journal of Education, Health and Sport, 2016; vol. 6, 10, pp. 754-771. DOI: http://dx.doi.org/10.5281/zenodo.258831.

[16] P. Vogt, Ch. Alexander Rüst, Th. Rosemann, R. Lepers, B. Knechtle. Analysis of $10 \mathrm{~km}$ swimming performance of elite male and female open-water swimmers. Springerplus. 2013, vol. 2, pp. 603. DOI: 10.1186/2193-1801-2-603. URL: https://springerplus.springeropen.com/articles/10.1186/2193-1801-2603.

[17] M.A. Zinng, C.A. Rüst, T. Rosemann, R. Lepers, B. Knechtle. Analysis of swimming performance in FINA world cup long-distance open water races. Extreme Physiology and Medicine. vol. 3, 1. URL: https://doi.org/10.1186/2046-7648-3-2.

[18] Evgeniya A. Raspopova, Irina V. Chebotareva. Physical development of young 11-13-years-old swimmers and divers. The Russian Journal of Physical Education and Sport. 2019, 14(1), pp. 29-33. DOI: 10.14526/2070-4798-2019-14-1-33-37.

[19] M.A. Zinng, C.A. Rüst, T. Rosemann, R. Lepers, B. Knechtle. Analysis of swimming performance in FINA world cup long-distance open water races. Extreme Physiology \& Medicine. 2014, vol. 3, 2. URL: http://www.extremephysiolmed.com/content/3/1/2. 